\title{
The Reduced Neighborhood Topological Indices and RNM - Polynomial for the Treatment of COVID-19
}

\author{
Anwar Saleh ${ }^{1(\mathbb{D})}$, Sophia Shalini G. B. ${ }^{2, *(\mathbb{D})}$, Dhananjayamurthy B. V. ${ }^{3(\mathbb{D})}$ \\ Department of Mathematics, Faculty of Science, University of Jeddah, Saudi Arabia; math.msfs@gmail.com (A.S.); \\ Department of Mathematics, Mount Carmel College, Bangalore-58, India; sofi.saranya920@ gmail.com (S.S.G.B.); \\ Department of Mathematics, Nitte Meenakshi Institute of Technology, Yelahanka, Bangalore-64; dmgyashas@gmail.com \\ (D.B.V.); \\ * Correspondence: sofi.saranya920@gmail.com;
}

Received: 19.11.2020; Revised: 14.12.2020; Accepted: 15.12.2020; Published: 18.12.2020

\begin{abstract}
A graphical index is a numeric value corresponding to a structurally invariant graph, and in molecular graph theory, these invariants are known as topological indices. In the field of Chemical and Medical Sciences, the topological indices are used to study the chemical, biological, medical, and pharmaceutical features of drugs. Concerning the previous deadly diseases, the COVID-19 pandemic has been considered the biggest life-threatening issue that modern medicines have ever tackled. COVID-19 is immedicable, and even the existing treatments are only helping a certain group of sufferers. Scientists have tested available antiviral agents and got a favorable impact on recovering from the pandemic. Some of these antiviral agents are remdesivir, chloroquine, hydroxychloroquine, theaflavin, and dexamethasone. Keeping given the importance of topological indices in the study of pharmaceutical and chemical drugs, in this paper, we calculate the reduced neighborhood topological indices and RNM-polynomial of some of the antiviral agents remdesivir, chloroquine, hydroxychloroquine, theaflavin, and dexamethasone. The results thus obtained may be useful for finding new medicine and vaccine for the treatment of COVID-19.
\end{abstract}

Keywords: COVID-19; remdesivir; chloroquine; hydroxychloroquine; theaflavin; dexamethasone; reduced neighborhood topological index.

(C) 2020 by the authors. This article is an open-access article distributed under the terms and conditions of the Creative Commons Attribution (CC BY) license (https://creativecommons.org/licenses/by/4.0/).

\section{Introduction}

In the period of dynamic technical development, many drugs and pharmaceutical techniques have been emerging every year, which requires a massive amount of work to examine the biological and chemical properties of these drugs. Also, many experiments have to be done on these new drugs to find out the side effects and benefits on the human body. These heavy work loaded experiments in laboratories may affect impoverished countries, especially Africa and Southeast Asia. During the initial stages of chemical experiments, the scientists have compared the compounds' structure and their experimental values and pointed out that they are closely related [1,2]. Calculating the properties of the compounds' molecular structure in terms of topological indices, the pharmaceutical and medical scholars may find them useful in studying the medicinal properties of the drugs.

In medical mathematics modeling, the structure of medicine is considered an undirected graph, where the vertices and edges are considered to be atoms and the chemical bonds, respectively. The information pertaining to physiochemical properties and the biological activities of the molecular graph of compounds are important in pharmaceutical drug design. 
These properties can be anticipated without any use of laboratories but by a conventional aid of chemical graph theory known as the topological index. A graphical index is a numeric value corresponding to a structurally invariant graph. In molecular graph theory, these invariants are known as topological indices. The first and second Zagreb indices are extensively studied among the various classes of topological indices. They have many applications in molecular graph theory. The Zagreb indices play a vital role in the theory of total $\pi$-electron energy of alternant hydrocarbons. Gutman and Trinajstic introduced the first and second Zagreb indices in 1972 [3].

With reference to the previous deadly diseases, the COVID-19 pandemic has been considered to be the biggest life-threatening issue that modern medicines have ever tackled. Scientists and doctors have been working tirelessly to find the drugs that may save the sufferers and may even protect them from getting affected. The first case was identified in Wuhan, Hubei, China, in December 2019, which resulted in an ongoing pandemic. As of 26th August 2020, more than 24 million reported resulting in 819,000 deaths, and 16,620,943 have been recovered across 188 countries and territories (from world meter information). COVID-19 is immedicable, and even the existing treatments are only helping a certain group of sufferers. No treatment has been fully licensed by the food and the drug administration agency for COVID19.

Scientists have tested some of the available antiviral agents and positively impacted recovering from pandemic by using remdesivir, chloroquine, hydroxychloroquine, theaflavin, and dexamethasone. The first drug to get emergency approval from food and drug administration for the use of COVID-19 is remdesivir. It ceases the reproduction of the virus. This drug was initially used as an antiviral agent for Hepatitis $\mathrm{C}$ and Ebola. From the preliminary trials, it has been observed that the drug can reduce the recovery time of the COVID-19 sufferers from 15 days to 11 days. In 1930s the, German scientists incorporated chloroquine as a drug against malaria. In 1946, the scientists invented the less toxic version of chloroquine called hydroxychloroquine. Later, the drug was approved for other diseases. During the initial stages of the deadly pandemic, scientists have found that both chloroquine and hydroxychloroquine can control the virus from reproducing the cells [4]. Initial reports from France and China have proposed that by giving chloroquine or hydroxychloroquine, the COVID-19 sufferers are recovered quickly. Theaflavin is a polyphenol chemical found in black tea that acts as an antiviral agent in treating influenza A, B, and hepatitis $\mathrm{C}$ virus. Lung et al. [5] have suggested that this drug can be used as a primary factor in producing a drug against COVID-19. British researchers on 17th July 2020 published that dexamethasone improves the immune response of the COVID-19 positives. The researchers' collaborative recovery group has found that this drug reduces the death rate of patients on ventilators by one-third and for the patients on oxygen by one-fifth. Nevertheless, it may be less effective and even may be harmful to patients at an earlier stage of COVID-19 infections [6]. However, in the COVID-19 treatment guidelines, the National Institutes of Health recommends using dexamethasone in patients with COVID-19 who are on a ventilator or are receiving supplemental oxygen.

Sourav Mondal, Nilanjan De, Anita Pal investigated the degree-based and neighborhood degree sum based topological indices for some of the antiviral drugs by using the polynomial approach [7]. Also, V.R. Kulli [8] calculated the Revan Indices for some of the antiviral drugs. For applied topological indices, refer [9-20]. In communication with the first author, he has some more work about the different types of reduced neighborhood indices that will appear soon. Keeping the importance of topological indices in the study of pharmaceutical 
and chemical drugs, in this paper, we calculate the reduced neighborhood first and second Zagreb indices, reduced neighborhood forgotten topological index, hyper reduced neighborhood topological index, modified reduced neighborhood version of first Zagreb index and modified reduced neighborhood version of first Zagreb index and forgotten topological index of some of the antiviral agents remdesivir, chloroquine, hydroxychloroquine, theaflavin and dexamethasone without considering the hydrogen bond of these compounds, since the vertices corresponding to the hydrogen atom does not make any meaning to the isomorphism of graphs. Also, the RNM-polynomial is introduced, and surface representation of the RNMpolynomial has been depicted.

\section{Materials and Methods}

Our main results include the properties and calculations of the chemical compounds used for the treatment of COVID-19 in terms of reduced neighborhood topological indices, particularly remdesivir, chloroquine, hydroxychloroquine, theaflavin, and dexamethasone. We evaluate the $R N M$ - polynomial of these structures with 3D graphical representation using MATLAB 2017a.

\section{Results and Discussion}

We divide this section into two subsections with the definitions and computational results.

\subsection{Reduced neighborhood topological indices and RNM-polynomial}

In communication with the first author, he has some more work about the different types of reduced neighborhood indices that will appear soon. The types of reduced neighborhood indices were defined as follows:

\subsubsection{Definition.}

Let $G=(V, E)$ be a simple graph with $|V(G)|=n$ and $|E(G)|=n$. The $N$-degree of a vertex $u$ is defined as the sum of the degree of the vertices in the open neighborhood of $u$. That is,

$$
d_{N}(u)=\sum_{v \in N(u)} d(v)
$$

The reduced neighborhood degree of a vertex $u$ is defined as

$$
d_{N^{\prime}}(u)=\sum_{v \in N(u)}(d(v)-1)
$$

\subsubsection{Definition.}

Let $G=(V, E)$ be a simple graph with $|V(G)|=n$ and $|E(G)|=n$. Then the reduced neighborhood first Zagreb index and second Zagreb index denoted by $R N M_{1}(G)$ and $R M_{2}(G)$ respectively, are defined as,

$$
R N M_{1}(G)=\sum_{u \in V(G)} d_{N^{\prime}}(u)^{2} \text { and } R N M_{2}(G)=\sum_{u v \in E(G)} d_{N^{\prime}}(u) d_{N^{\prime}}(v) .
$$




\subsubsection{Definition.}

We define the modified, reduced neighborhood version of the first Zagreb index denoted by $R N M_{1}^{*}(G)$ as follows:

$$
\operatorname{RNM}_{1}^{*}(G)=\sum_{u v \in E(G)}\left[d_{N^{\prime}}(u)+d_{N^{\prime}}(v)\right] .
$$

\subsubsection{Definition.}

Let $G=(V, E)$ be a simple graph with $|V(G)|=n$ and $|E(G)|=n$. Then the forgotten, reduced neighborhood index denoted by $R N M_{3}(G)$ is defined as

$$
\operatorname{RNM}_{3}(G)=\sum_{u \in V(G)} d_{N^{\prime}}(u)^{3} .
$$

\subsubsection{Definition.}

We define the modified, reduced neighborhood version of the forgotten topological index denoted by $\operatorname{RNM}_{3}^{*}(G)$ as follows:

$$
R N M_{3}^{*}(G)=\sum_{u v \in E(G)}\left[d_{N^{\prime}}(u)^{2}+d_{N^{\prime}}(v)^{2}\right] .
$$

\subsubsection{Definition}

Let $G=(V, E)$ be a simple graph with $|V(G)|=n$ and $|E(G)|=n$. Then the hyper reduced neighborhood index denoted by $\operatorname{HRN}(G)$ is defined as

$$
\operatorname{HRN}(G)=\sum_{u v \in E(G)}\left[d_{N^{\prime}}(u)+d_{N^{\prime}}(v)\right]^{2} .
$$

\subsubsection{Definition.}

Let the edge partition of the graph $G$ be defined as

$$
R_{(i, j)}(G)=\left\{u v \in E(G): d_{N^{\prime}}(u)=i \text { and } d_{N^{\prime}}(v)=j\right\} \text {. }
$$

The maximum degree $\Delta_{N^{\prime}}(G)$ is defined as $\Delta_{N^{\prime}}=\max \left\{d_{N^{\prime}}(v): v \in V(G)\right\}$.

The minimum degree $\delta_{N^{\prime}}(G)$ is defined as $\delta_{N^{\prime}}=\min \left\{d_{N^{\prime}}(v): v \in V(G)\right\}$.

\subsubsection{Definition.}

Let $G$ be a graph and let $R_{i j}(G), i, j \geq 1$, be the number of edges $e=u v$ of $G$ such that $\left\{d_{u}(G), d_{v}(G)\right\}=\{i, j\}$. Let $R_{i j}^{\prime}=\left|R_{(i, j)}\right|$. We introduce the $R N M$-polynomial of a graph $G$ as

$$
R N M(G, x, y)=\sum_{\delta_{N^{\prime} \leq i \leq j \leq \Delta_{N}}} R_{i j}^{\prime} x^{i} y^{j}
$$

3.2. The reduced neighborhood topological indices and RNM - polynomial for the treatment of COVID-19.

\subsubsection{Remdesivir.}

Let $G$ be a molecular graph of remdesivir, as shown in Figure 1. The graph $G$ has 41 vertices and 43 edges. 


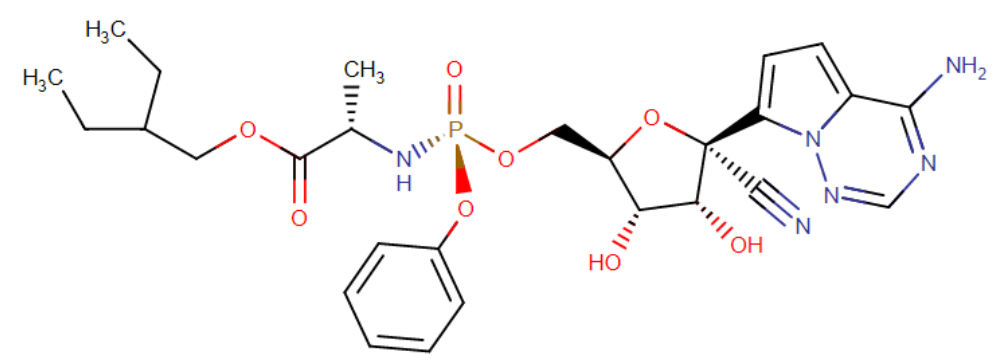

Figure 1. Chemical structure of remdesivir.

Theorem 1. Let $G$ be a molecular graph of remdesivir. Then

$$
\begin{aligned}
R N M(G, x, y)= & 2 x y^{2}+9 x^{2} y^{3}+9 x^{3} y^{3}+7 x^{3} y^{5}+2 x^{2} y^{2}+3 x^{3} y^{4}+x^{4} y^{4}+ \\
& x^{2} y^{4}+x^{2} y^{5}+2 x^{5} y^{4}+2 x^{5} y^{6}+3 x^{5} y^{5}+x^{6} y^{3}+x y^{5} .
\end{aligned}
$$

Proof. Let $R_{(i, j)}$ be the set of different types of edges as defined in Definition 3.1.7 and let $R_{i j}^{\prime}$ be the number of edges in $R_{(i, j)}$. From Figure 1 , it can be calculated that $R_{12}^{\prime}=2, R_{23}^{\prime}=$ $9, R_{33}^{\prime}=9, R_{35}^{\prime}=7, R_{22}^{\prime}=2, R_{34}^{\prime}=3, R_{44}^{\prime}=1, R_{24}^{\prime}=1, R_{25}^{\prime}=1, R_{54}^{\prime}=2, R_{56}^{\prime}=2, R_{55}^{\prime}=$ $3, R_{63}^{\prime}=1, R_{15}^{\prime}=1$. The $R N M-$ polynomial of $G$ is obtained as follows:

$$
\begin{aligned}
& R N M(G, x, y)=\sum_{\delta_{N \prime \leq i \leq j \leq \Delta_{N}}} R_{i j}^{\prime} x^{i} y^{j} \\
& =R_{12}^{\prime} x y^{2}+R_{23}^{\prime} x^{2} y^{3}+R_{33}^{\prime} x^{3} y^{3}+R_{35}^{\prime} x^{3} y^{5}+R_{22}^{\prime} x^{2} y^{2}+R_{34}^{\prime} x^{3} y^{4}+R_{44}^{\prime} x^{4} y^{4} \\
& +R_{24}^{\prime} x^{2} y^{4}+R_{25}^{\prime} x^{2} y^{5}+R_{54}^{\prime} x^{5} y^{4}+R_{56}^{\prime} x^{5} y^{6}+R_{55}^{\prime} x^{5} y^{5}+R_{63}^{\prime} x^{6} y^{3}+R_{15}^{\prime} x y^{5} \\
& =2 x y^{2}+9 x^{2} y^{3}+9 x^{3} y^{3}+7 x^{3} y^{5}+2 x^{2} y^{2}+3 x^{3} y^{4}+x^{4} y^{4}+x^{2} y^{4} \\
& +x^{2} y^{5}+2 x^{5} y^{4}+2 x^{5} y^{6}+3 x^{5} y^{5}+x^{6} y^{3}+x y^{5} .
\end{aligned}
$$

In Figure 2, we show the plot of RNM - polynomial of remdesivir.

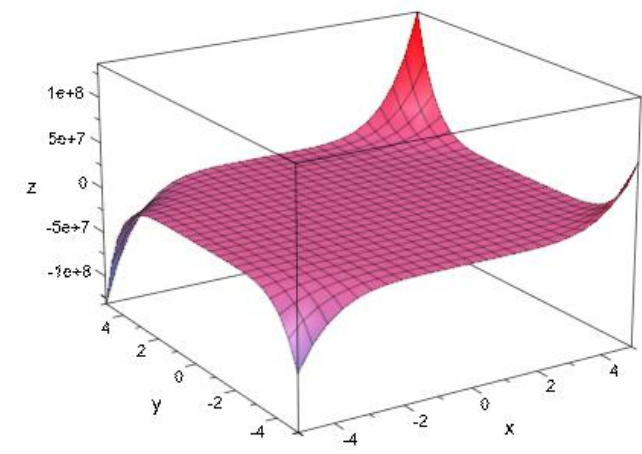

Figure 2. The plot of $R N M-$ polynomial of remdesivir.

Theorem 2. Let $G$ be a molecular graph of remdesivir. Then

$$
\begin{aligned}
& R N M_{1}(G)=450, \operatorname{RNM}_{2}(G)=556, R N M_{3}(G)=1806, \operatorname{HRN}(G)=2164, \\
& \operatorname{RNM}_{1}^{*}(G)=296, \operatorname{RNM}_{3}^{*}(G)=1124 .
\end{aligned}
$$

Proof. The reduced neighborhood vertex degrees of $G$ are listed in Table 1 . 
Table 1. The reduced neighborhood vertex degree of $G$.

\begin{tabular}{c|c|c|c|c|c|c}
$\boldsymbol{d}_{\boldsymbol{N}^{\prime}}(\boldsymbol{v})$ & 1 & 2 & 3 & 4 & 5 & 6 \\
\hline$\left|\boldsymbol{d}_{\boldsymbol{N}^{\prime}}(\boldsymbol{v})\right|$ & 3 & 11 & 16 & 3 & 7 & 1
\end{tabular}

Using the Definitions 3.1.2, 3.1.4 and Table 1, we have

$$
\begin{aligned}
\operatorname{RNM}_{1}(G) & =\sum_{u \in V(G)} d_{N^{\prime}}(u)^{2} \\
& =3.1^{2}+11.2^{2}+16.3^{2}+3.4^{2}+7.5^{2}+1.6^{2} \\
& =450 . \\
\operatorname{RNM}_{3}(G) & =\sum_{u \in V(G)} d_{N^{\prime}}(u)^{3} \\
& =3.1^{3}+11.2^{3}+16.3^{3}+3.4^{3}+7.5^{3}+1.6^{3} \\
& =1806 .
\end{aligned}
$$

Using the edge partition values from Theorem 1 and Definitions 3.1.2, 3.1.6, we have

$$
\begin{aligned}
R N M_{2}(G)= & \sum_{u v \in E(G)} d_{N^{\prime}}(u) d_{N^{\prime}}(v) \\
=2(1.2)+9(2.3)+9(3.3)+7(3.5)+2(2.2)+3(3.4)+1(4.4)+1(2.4)+1(2.5) & \\
& +2(5.4)+2(5.6)+3(5.5)+1(6.3)+1(1.5) \\
= & 556 . \\
H R N(G)= & \sum_{u v \in E(G)}\left[d_{N^{\prime}}(u)+d_{N^{\prime}}(v)\right]^{2} \\
= & 2(1+2)^{2}+9(2+3)^{2}+9(3+3)^{2}+7(3+5)^{2}+2(2+2)^{2}+3(3+4)^{2} \\
& +1(4+4)^{2}+1(2+4)^{2}+1(2+5)^{2}+2(5+4)^{2}+2(5+6)^{2}+3(5+5)^{2} \\
& +1(6+3)^{2}+1(1+5)^{2} \\
= & 2164 .
\end{aligned}
$$

Using the Definitions 3.1.3,3.1.5 and Table 1, we have

$$
\begin{aligned}
R N M_{1}^{*}(G) & =\sum_{u v \in E(G)}\left[d_{N^{\prime}}(u)+d_{N^{\prime}}(v)\right] \\
& =2(1+2)+9(2+3)+9(3+3)+7(3+5)+2(2+2)+3(3+4)+1(4+4) \\
& +1(2+4)+1(2+5)+2(5+4)+2(5+6)+3(5+5)+1(6+3)+1(1+5) \\
& =296 .
\end{aligned}
$$$$
R N M_{3}^{*}(G)=\sum_{u v \in E(G)}\left[d_{N^{\prime}}(u)^{2}+d_{N^{\prime}}(v)^{2}\right]
$$$$
=2\left(1^{2}+2^{2}\right)+9\left(2^{2}+3^{2}\right)+9\left(3^{2}+3^{2}\right)+7\left(3^{2}+5^{2}\right)+2\left(2^{2}+2^{2}\right)
$$$$
+3\left(3^{2}+4^{2}\right)+1\left(4^{2}+4^{2}\right)+1\left(2^{2}+4^{2}\right)+1\left(2^{2}+5^{2}\right)+2\left(5^{2}+4^{2}\right)+2\left(5^{2}+6^{2}\right)
$$ 


$$
\begin{aligned}
& +3\left(5^{2}+5^{2}\right)+1\left(6^{2}+3^{2}\right)+1\left(1^{2}+5^{2}\right) \\
& =1124 .
\end{aligned}
$$

\subsubsection{Chloroquine.}

Let $G$ be a molecular graph of chloroquine as shown in Figure 3. The graph $G$ has 22 vertices and 23 edges.<smiles>CCN(CC)CCCC(C)Nc1ccnc2cc(Cl)ccc12</smiles>

Figure 3. Chemical structure of chloroquine.

Theorem 3. Let $G$ be a molecular graph of chloroquine. Then

$$
R N M(G, x, y)=2 x^{2} y^{2}+8 x^{2} y^{3}+2 x^{2} y^{4}+2 x^{3} y^{3}+2 x^{4} y^{4}+2 x^{3} y^{4}+x^{3} y^{5}+2 x^{5} y^{4}+2 x y^{2} \text {. }
$$

Proof. Let $R_{(i, j)}$ be the set of different types of edges as defined in Definition 3.1.7 and let $R_{i j}^{\prime}$ be the number of edges in $R_{(i, j)}$. From Figure 3, it can be calculated that $R_{22}^{\prime}=2, R_{23}^{\prime}=$ $8, R_{24}^{\prime}=2, R_{33}^{\prime}=2, R_{44}^{\prime}=2, R_{34}^{\prime}=2, R_{35}^{\prime}=1, R_{54}^{\prime}=2, R_{12}^{\prime}=2$. The $R N M$-polynomial of $G$ is obtained as follows:

$$
\begin{aligned}
R N M(G, x, y)= & \sum_{\delta_{N^{\prime} \leq i \leq j \leq \Delta_{N^{\prime}}}} R_{i j}^{\prime} x^{i} y^{j} \\
= & R_{22}^{\prime} x^{2} y^{2}+R_{23}^{\prime} x^{2} y^{3}+R_{24}^{\prime} x^{2} y^{4}+R_{33}^{\prime} x^{3} y^{3}+R_{44}^{\prime} x^{4} y^{4}+R_{34}^{\prime} x^{3} y^{4} \\
& +R_{35}^{\prime} x^{3} y^{5}+R_{54}^{\prime} x^{5} y^{4}+R_{12}^{\prime} x y^{2} \\
= & 2 x^{2} y^{2}+8 x^{2} y^{3}+2 x^{2} y^{4}+2 x^{3} y^{3}+2 x^{4} y^{4}+2 x^{3} y^{4} \\
& +1 x^{3} y^{5}+2 x^{5} y^{4}+2 x y^{2} .
\end{aligned}
$$

In Figure 4, we show the plot of $R N M$ - polynomial of chloroquine.

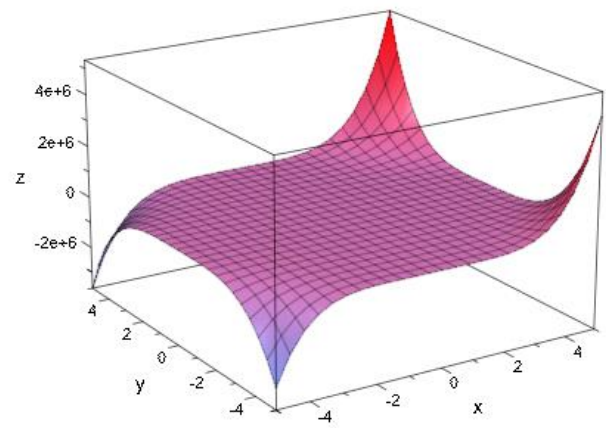

Figure 4. The plot of $R N M$ - polynomial of chloroquine.

Theorem 4. Let $G$ be a molecular graph of chloroquine. Then $R N M_{1}(G)=186, R N M_{2}(G)=205, R N M_{3}(G)=636, H R N(G)=846, R N M_{1}^{*}(G)=134$, 
$\operatorname{RNM}_{3}^{*}(G)=436$.

Proof. The reduced neighborhood vertex degrees of $G$ are listed in Table 2.

Table 2. The reduced neighborhood vertex degree of $G$.

\begin{tabular}{c|c|c|c|c|c}
$\boldsymbol{d}_{\boldsymbol{N}^{\prime}}(\boldsymbol{v})$ & $\mathbf{1}$ & $\mathbf{2}$ & $\mathbf{3}$ & $\mathbf{4}$ & $\mathbf{5}$ \\
\hline$\left|\boldsymbol{d}_{\boldsymbol{N}^{\prime}}(\boldsymbol{v})\right|$ & 2 & 8 & 7 & 4 & 1
\end{tabular}

Using the Definitions 3.1.2, 3.1.4 and Table 2, we have

$$
\begin{aligned}
\operatorname{RNM}_{1}(G) & =\sum_{u \in V(G)} d_{N^{\prime}}(u)^{2} \\
& =2.1^{2}+8.2^{2}+7.3^{2}+4.4^{2}+1.5^{2} \\
& =186 . \\
\operatorname{RNM}_{3}(G) & =\sum_{u \in V(G)} d_{N^{\prime}}(u)^{3} \\
& =2.1^{3}+8.2^{3}+7.3^{3}+4.4^{3}+1.5^{3} \\
& =636 .
\end{aligned}
$$

Using the edge partition values from Theorem 3 and Definitions 3.1.2, 3.1.6, we have

$$
\begin{aligned}
R N M_{2}(G)= & \sum_{u v \in E(G)} d_{N^{\prime}}(u) d_{N^{\prime}}(v) \\
= & 2(2.2)+8(2.3)+2(2.4)+2(3.3)+2(4.4)+2(3.4)+1(3.5)+2(5.4)+ \\
& 2(1.2) \\
= & 205 . \\
H R N(G)= & \sum_{u v \in E(G)}\left[d_{N^{\prime}}(u)+d_{N^{\prime}}(v)\right]^{2} \\
= & 2(2+2)^{2}+8(2+3)^{2}+2(2+4)^{2}+2(3+3)^{2}+2(4+4)^{2}+2(3+4)^{2} \\
& +1(3+5)^{2}+2(5+4)^{2}+2(1+2)^{2} \\
= & 846 .
\end{aligned}
$$

Using the Definitions 3.1.3,3.1.5 and Table 2, we have

$$
\begin{aligned}
R N M_{1}^{*}(G)= & \sum_{u v \in E(G)}\left[d_{N^{\prime}}(u)+d_{N^{\prime}}(v)\right] \\
= & 2(2+2)+8(2+3)+2(2+4)+2(3+3)+2(4+4)+2(3+4)+ \\
& 1(3+5)+2(5+4)+2(1+2) \\
= & 134 .
\end{aligned}
$$$$
\operatorname{RNM}_{3}^{*}(G)=\sum_{u v \in E(G)}\left[d_{N^{\prime}}(u)^{2}+d_{N^{\prime}}(v)^{2}\right]
$$$$
=2\left(2^{2}+2^{2}\right)+8\left(2^{2}+3^{2}\right)+2\left(2^{2}+4^{2}\right)+2\left(3^{2}+3^{2}\right)+2\left(4^{2}+4^{2}\right)
$$$$
+2\left(3^{2}+4^{2}\right)+1\left(3^{2}+5^{2}\right)+2\left(5^{2}+4^{2}\right)+2\left(1+2^{2}\right)
$$ 


$$
=436 \text {. }
$$

\subsubsection{Hydroxychloroquine.}

Let $G$ be a molecular graph of hydroxychloroquine, as shown in Figure 5. The graph $G$ has 23 vertices and 24 edges.

Theorem 5. Let $G$ be a molecular graph of hydroxychloroquine. Then $R N M(G, x, y)=2 x^{2} y^{2}+7 x^{2} y^{3}+3 x^{3} y^{3}+2 x^{2} y^{4}+2 x^{4} y^{4}+2 x^{3} y^{4}+x^{3} y^{5}+2 x^{5} y^{4}+x y^{2}+x y^{3}+x y$.

Proof. Let $R_{(i, j)}$ be the set of different types of edges as defined in Definition 3.1.7 and let $R_{i j}^{\prime}$ be the number of edges in $R_{(i, j)}$. From Figure 5, it can be calculated that $R_{22}^{\prime}=$ $2, R_{23}^{\prime}=7, R_{24}^{\prime}=2, R_{33}^{\prime}=3, R_{44}^{\prime}=2, R_{34}^{\prime}=2, R_{35}^{\prime}=1, R_{54}^{\prime}=2, R_{12}^{\prime}=1, R_{13}^{\prime}=1, R_{11}^{\prime}=$ 1. The $R N M$ - polynomial of $G$ is obtained as follows:

$$
\begin{aligned}
R N M(G, x, y)= & \sum_{\delta_{N^{\prime} \leq i \leq j \leq \Delta_{N^{\prime}}}} R_{i j}^{\prime} x^{i} y^{j} \\
= & R_{22}^{\prime} x^{2} y^{2}+R_{23}^{\prime} x^{2} y^{3}+R_{24}^{\prime} x^{2} y^{4}+R_{33}^{\prime} x^{3} y^{3}+R_{44}^{\prime} x^{4} y^{4}+R_{34}^{\prime} x^{3} y^{4} \\
& +R_{35}^{\prime} x^{3} y^{5}+R_{54}^{\prime} x^{5} y^{4}+R_{12}^{\prime} x y^{2}+R_{13}^{\prime} x y^{3}+R_{11}^{\prime} x y \\
= & 2 x^{2} y^{2}+7 x^{2} y^{3}+2 x^{2} y^{4}+3 x^{3} y^{3}+2 x^{4} y^{4}+2 x^{3} y^{4} \\
& +1 x^{3} y^{5}+2 x^{5} y^{4}+x y^{2}+x y^{3}+x y .
\end{aligned}
$$<smiles>CCN(CCO)CCCC(C)Nc1ccnc2cc(Cl)ccc12</smiles>

Figure 5. Chemical structure of hydroxychloroquine.

In Figure 6, we show the plot of $R N M$-polynomial of hydroxychloroquine.

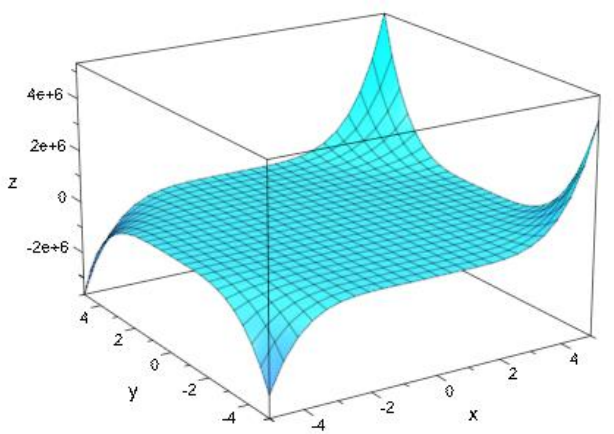

Figure 6. The plot of $R N M$ - polynomial of hydroxychloroquine.

Theorem 6. Let $G$ be a molecular graph of hydroxychloroquine. Then 
$R N M_{1}(G)=192, R_{N} M_{2}(G)=210, R N M_{3}(G)=656, H R N(G)=868, \operatorname{RNM}_{1}^{*}(G)=138$, $\operatorname{RNM}_{3}^{*}(G)=448$.

Proof. The reduced neighborhood vertex degrees of $G$ are listed in Table 3.

Table 3. The reduced neighborhood vertex degree of $G$.

\begin{tabular}{c|c|c|c|c|c}
$\boldsymbol{d}_{\boldsymbol{N}^{\prime}}(\boldsymbol{v})$ & 1 & 2 & 3 & 4 & 5 \\
\hline$\left|\boldsymbol{d}_{\boldsymbol{N}^{\prime}}(\boldsymbol{v})\right|$ & 3 & 7 & 8 & 4 & 1
\end{tabular}

Using the Definitions 3.1.2, 3.1.4, and Table 3, we have

$$
\begin{aligned}
\operatorname{RNM}_{1}(G) & =\sum_{u \in V(G)} d_{N^{\prime}}(u)^{2} \\
& =3.1^{2}+7.2^{2}+8 \cdot 3^{2}+4.4^{2}+1.5^{2} \\
& =192 . \\
\operatorname{RNM}_{3}(G) & =\sum_{u \in V(G)} d_{N^{\prime}}(u)^{3} \\
& =3.1^{3}+7.2^{3}+8.3^{3}+4.4^{3}+1.5^{3} \\
& =656 .
\end{aligned}
$$

Using the edge partition values from Theorem 5 and Definitions 3.1.2, 3.1.6, we have

$$
\begin{aligned}
R N M_{2}(G)= & \sum_{u v \in E(G)} d_{N^{\prime}}(u) d_{N^{\prime}}(v) \\
= & 2(2.2)+7(2.3)+2(2.4)+3(3.3)+2(4.4)+2(3.4)+1(3.5)+2(5.4)+1(1.2) \\
& +1(1.3)+1 \\
= & 210 . \\
H R N(G)= & \sum_{u v \in E(G)}\left[d_{N^{\prime}}(u)+d_{N^{\prime}}(v)\right]^{2} \\
= & 2(2+2)^{2}+7(2+3)^{2}+2(2+4)^{2}+3(3+3)^{2}+2(4+4)^{2}+2(3+4)^{2} \\
& +1(3+5)^{2}+2(5+4)^{2}+1(1+2)^{2}+1(1+3)^{2}+1(1+1)^{2} \\
= & 868 .
\end{aligned}
$$

Using the Definitions 3.1.3, 3.1.5 and Table 3, we have

$$
\begin{aligned}
R N M_{1}^{*}(G) & =\sum_{u v \in E(G)}\left[d_{N^{\prime}}(u)+d_{N^{\prime}}(v)\right] \\
= & 2(2+2)+7(2+3)+2(2+4)+3(3+3)+2(4+4)+2(3+4)+1(3+5) \\
& +2(5+4)+(1+2)+(1+3)+(1+1) \\
& =138 .
\end{aligned}
$$




$$
\begin{aligned}
R N M_{3}^{*}(G) & =\sum_{u v \in E(G)}\left[d_{N^{\prime}}(u)^{2}+d_{N^{\prime}}(v)^{2}\right] \\
& =2\left(2^{2}+2^{2}\right)+7\left(2^{2}+3^{2}\right)+2\left(2^{2}+4^{2}\right)+3\left(3^{2}+3^{2}\right)+2\left(4^{2}+4^{2}\right) \\
& +2\left(3^{2}+4^{2}\right)+1\left(3^{2}+5^{2}\right)+2\left(5^{2}+4^{2}\right)+\left(1+2^{2}\right)+\left(1+3^{2}\right)+(1+1) \\
& =448 .
\end{aligned}
$$

\subsubsection{Theaflavin.}

Let $G$ be a molecular graph of hydroxychloroquine, as shown in Figure 7. The graph $G$ has 41 vertices and 46 edges.

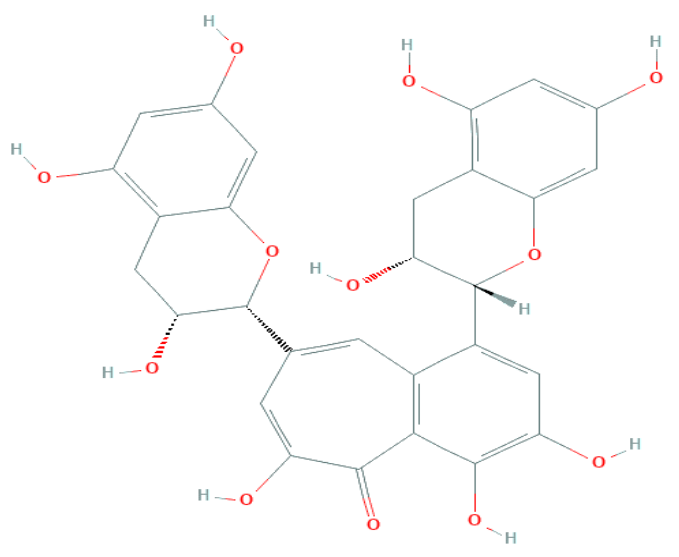

Figure 7. Chemical structure of theaflavin.

Theorem 7. Let $G$ be a molecular graph of theaflavin. Then

$$
\begin{aligned}
R N M(G, x, y)= & 2 x^{2} y^{2}+6 x^{2} y^{3}+6 x^{2} y^{4}+4 x^{3} y^{5}+6 x^{4} y^{4}+9 x^{5} y^{4}+8 x^{3} y^{4} \\
& +2 x^{3} y^{4}+2 x^{5} y^{5}+2 x^{4} y^{6}+x^{5} y^{6}
\end{aligned}
$$

Proof. Let $R_{(i, j)}$ be the set of different types of edges as defined in Definition 3.1.7 and let $R_{i j}^{\prime}$ be the number of edges in $R_{(i, j)}$. From Figure 8 , it can be calculated that $R_{22}^{\prime}=$ $2, R_{23}^{\prime}=6, R_{24}^{\prime}=6, R_{35}^{\prime}=4, R_{44}^{\prime}=6, R_{54}^{\prime}=9, R_{34}^{\prime}=8, R_{55}^{\prime}=2, R_{46}^{\prime}=2, R_{56}^{\prime}=1$. The $R N M$ - polynomial of $G$ is obtained as follows:

$$
\begin{aligned}
& R N M(G, x, y)=\sum_{\delta_{N \prime \leq i \leq j \leq \Delta_{N^{\prime}}}} R_{i j}^{\prime} x^{i} y^{j} \\
& =R_{22}^{\prime} x^{2} y^{2}+R_{23}^{\prime} x^{2} y^{3}+R_{24}^{\prime} x^{2} y^{4}+R_{35}^{\prime} x^{3} y^{5}+R_{44}^{\prime} x^{4} y^{4}+R_{54}^{\prime} x^{5} y^{4}+R_{34}^{\prime} x^{3} y^{4} \\
& \quad+R_{55}^{\prime} x^{5} y^{5}+R_{46}^{\prime} x^{4} y^{6}+R_{56}^{\prime} x^{5} y^{6} \\
& =2 x^{2} y^{2}+6 x^{2} y^{3}+6 x^{2} y^{4}+4 x^{3} y^{5}+6 x^{4} y^{4}+9 x^{5} y^{4}+8 x^{3} y^{4}+2 x^{5} y^{5} \\
& \quad+2 x^{4} y^{6}+x^{5} y^{6} .
\end{aligned}
$$

In Figure 8, we show the plot of $R N M$-polynomial of theaflavin. 


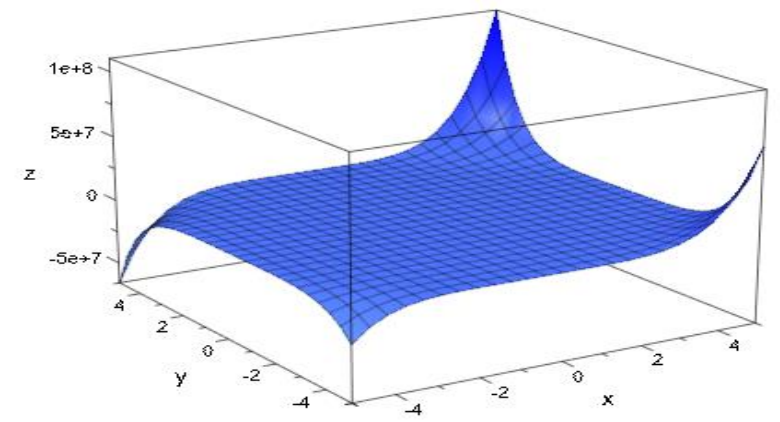

Figure 8. The plot of $R N M$ - polynomial of theaflavin.

Theorem 2.16. Let $G$ be a molecular graph of theaflavin. Then

$R N M_{1}(G)=554, R N M_{2}(G)=652, R N M_{3}(G)=2248, H R N(G)=2680$,

$R N M_{1}^{*}(G)=342, R N M_{3}^{*}(G)=1376$.

Proof. The reduced neighborhood vertex degrees of $G$ are listed in Table 4 .

Table 4. The reduced neighborhood vertex degree of $G$.

\begin{tabular}{c|c|c|c|c|c}
$\boldsymbol{d}_{\boldsymbol{N}^{\prime}}(\boldsymbol{v})$ & 2 & 3 & 4 & 5 & 6 \\
\hline$\left|\boldsymbol{d}_{\boldsymbol{N}^{\prime}}(\boldsymbol{v})\right|$ & 12 & 6 & 16 & 6 & 1
\end{tabular}

Using the Definitions 3.1.2, 3.1.4, and Table 4, we have

$$
\begin{aligned}
\operatorname{RNM}_{1}(G) & =\sum_{u \in V(G)} d_{N^{\prime}}(u)^{2} \\
& =12.2^{2}+6.3^{2}+16.4^{2}+6.5^{2}+1.6^{2} \\
& =544 . \\
\operatorname{RNM}_{3}(G) & =\sum_{u \in V(G)} d_{N^{\prime}}(u)^{3} \\
& =12.2^{3}+6.3^{3}+16.4^{3}+6.5^{3}+1.6^{3} \\
& =2248 .
\end{aligned}
$$

Using the edge partition values from Theorem 7 and Definitions 3.1.2, 3.1.6, we have

$$
\begin{aligned}
& R_{N} M_{2}(G)=\sum_{u v \in E(G)} d_{N^{\prime}}(u) d_{N^{\prime}}(v) \\
& =2(2.2)+6(2.3)+6(2.4)+4(3.5)+6(4.4)+9(5.4)+8(3.4)+2(5.5)+2(4.6) \\
& +1(5.6) \\
& =652 . \\
& H R N(G)=\sum_{u v \in E(G)}\left[d_{N^{\prime}}(u)+d_{N^{\prime}}(v)\right]^{2} \\
& =2(2+2)^{2}+6(2+3)^{2}+6(2+4)^{2}+4(3+5)^{2}+6(4+4)^{2}+9(5+4)^{2} \\
& \quad+8(3+4)^{2}+2(5+5)^{2}+2(4+6)^{2}+(5+6)^{2}
\end{aligned}
$$




$$
=2680 .
$$

Using the Definitions 3.1.3, 3.1.5 and Table 4, we have

$$
\begin{aligned}
R N M_{1}^{*}(G)= & \sum_{u v \in E(G)}\left[d_{N^{\prime}}(u)+d_{N^{\prime}}(v)\right] \\
= & 2(2+2)+6(2+3)+6(2+4)+4(3+5)+6(4+4)+9(5+4)+8(3+4) \\
& +2(5+5)+2(4+6)+(5+6) \\
= & 342 .
\end{aligned}
$$

$$
\begin{aligned}
\operatorname{RNM}_{3}^{*}(G) & =\sum_{u v \in E(G)}\left[d_{N^{\prime}}(u)^{2}+d_{N^{\prime}}(v)^{2}\right] \\
& =2\left(2^{2}+2^{2}\right)+6\left(2^{2}+3^{2}\right)+6\left(2^{2}+4^{2}\right)+4\left(3^{2}+5^{2}\right)+6\left(4^{2}+4^{2}\right) \\
& +9\left(5^{2}+4^{2}\right)+8\left(3^{2}+4^{2}\right)+2\left(5^{2}+5^{2}\right)+2\left(4^{2}+6^{2}\right)+\left(5^{2}+6^{2}\right) \\
& =1376 .
\end{aligned}
$$

\subsubsection{Dexamethasone.}

Let $G$ be a molecular graph of dexamethasone, as shown in Figure 9. The graph $G$ has 28 vertices and 31 edges.

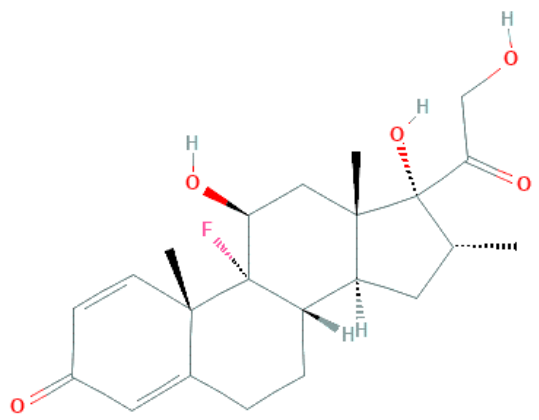

Figure 9. Chemical structure of dexamethasone.

Theorem 9. Let $G$ be a molecular graph of dexamethasone. Then

$$
\begin{aligned}
R N M(G, x, y) & =x y^{2}+x^{2} y^{2}+x^{2} y^{3}+5 x^{2} y^{4}+x^{3} y^{4}+2 x^{4} y^{5}+2 x^{4} y^{6}+2 x^{5} y^{6}+3 x^{3} y^{6} \\
& +x^{3} y^{3}+x^{5} y^{3}+3 x^{6} y^{7}+2 x^{6} y^{6}+2 x^{3} y^{7}+3 x^{4} y^{7}+x^{4} y^{4} .
\end{aligned}
$$

Proof. Let $R_{(i, j)}$ be the set of different types of edges as defined in Definition 3.1.7 and let $R_{i j}^{\prime}$ be the number of edges in $R_{(i, j)}$. From Figure 9, it can be calculated that $R_{12}^{\prime}=1, R_{22}^{\prime}=$ $1, R_{23}^{\prime}=1, R_{24}^{\prime}=5, R_{34}^{\prime}=1, R_{45}^{\prime}=2, R_{46}^{\prime}=2, R_{56}^{\prime}=2, R_{36}^{\prime}=3, R_{33}^{\prime}=1, R_{53}^{\prime}=1, R_{67}^{\prime}=$ $3, R_{66}^{\prime}=2, R_{37}^{\prime}=2, R_{47}^{\prime}=3, R_{44}^{\prime}=1$. The $R N M$-polynomial of $G$ is obtained as follows:

$$
\begin{aligned}
& R N M(G, x, y)=\sum_{\delta_{N^{\prime} \leq i \leq j \leq \Delta_{N^{\prime}}}} R_{i j}^{\prime} x^{i} y^{j} \\
& =R_{12}^{\prime} x y^{2}+R_{22}^{\prime} x^{2} y^{2}+R_{23}^{\prime} x^{2} y^{3}+R_{24}^{\prime} x^{2} y^{4}+R_{34}^{\prime} x^{3} y^{4}+R_{45}^{\prime} x^{4} y^{5}+R_{46}^{\prime} x^{4} y^{6} \\
& \quad+R_{56}^{\prime} x^{5} y^{6}+R_{36}^{\prime} x^{3} y^{6}+R_{33}^{\prime} x^{3} y^{3}+R_{53}^{\prime} x^{5} y^{3}+R_{67}^{\prime} x^{6} y^{7}+R_{66}^{\prime} x^{6} y^{6}+R_{37}^{\prime} x^{3} y^{7} \\
& \text { https://biointerfaceresearch.com/ }
\end{aligned}
$$




$$
\begin{aligned}
& +R_{47}^{\prime} x^{4} y^{7}+R_{44}^{\prime} x^{4} y^{4} \\
= & x y^{2}+x^{2} y^{2}+x^{2} y^{3}+5 x^{2} y^{4}+x^{3} y^{4}+2 x^{4} y^{5}+2 x^{4} y^{6}+2 x^{5} y^{6} \\
+ & 3 x^{3} y^{6}+x^{3} y^{3}+x^{5} y^{3}+3 x^{6} y^{7}+2 x^{6} y^{6}+2 x^{3} y^{7}+3 x^{4} y^{7}+x^{4} y^{4} .
\end{aligned}
$$

In Figure 2, we show the plot of RNM - polynomial of dexamethasone.

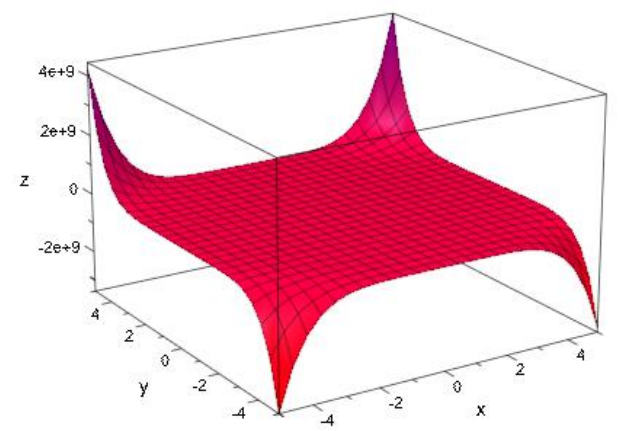

Figure 10. The plot of $R N M$ - polynomial of dexamethasone.

Theorem 10. Let $G$ be a molecular graph of dexamethasone. Then

$$
\begin{aligned}
& R N M_{1}(G)=476, R_{N} M_{2}(G)=630, R N M_{3}(G)=2422, H R N(G)=2648, \\
& R N M_{1}^{*}(G)=274, R_{N} M_{3}^{*}(G)=1388 .
\end{aligned}
$$

Proof. The reduced neighborhood vertex degrees of $G$ are listed in Table 5.

Table 5. The reduced neighborhood vertex degree of $G$.

\begin{tabular}{c|l|l|l|l|l|l|l}
$\boldsymbol{d}_{\boldsymbol{N}^{\prime}}(\boldsymbol{v})$ & 1 & 2 & 3 & 4 & 5 & 6 & 7 \\
\hline$\left|\boldsymbol{d}_{\boldsymbol{N}^{\prime}}(\boldsymbol{v})\right|$ & 1 & 6 & 7 & 6 & 2 & 4 & 2
\end{tabular}

Using the Definitions 3.1.2, 3.1.4, and Table 5, we have

$$
\begin{aligned}
\operatorname{RNM}_{1}(G) & =\sum_{u \in V(G)} d_{N^{\prime}}(u)^{2} \\
& =1+6.2^{2}+7.3^{2}+6.4^{2}+2.5^{2}+4.6^{2}+2.7^{2} \\
& =476 . \\
\operatorname{RNM}_{3}(G) & =\sum_{u \in V(G)} d_{N^{\prime}}(u)^{3} \\
& =1+6.2^{3}+7.3^{3}+6.4^{3}+2.5^{3}+4.6^{3}+2.7^{3} \\
& =2422 .
\end{aligned}
$$

Using the edge partition values from Theorem 9 and Definitions 3.1.2, 3.1.6, we have

$$
\begin{aligned}
\operatorname{RNM}_{2}(G)= & \sum_{u v \in E(G)} d_{N^{\prime}}(u) d_{N^{\prime}}(v) \\
= & (1.2)+(2.2)+(2.3)+5(2.4)+(3.4)+2(4.5)+2(4.6)+2(5.6)+3(3.6) \\
& +(3.3)+(5.3)+3(6.7)+2(6.6)+2(3.7)+3(4.7)+(4.4) \\
= & 630 .
\end{aligned}
$$




$$
\begin{aligned}
H R N(G)= & \sum_{u v \in E(G)}\left[d_{N^{\prime}}(u)+d_{N^{\prime}}(v)\right]^{2} \\
= & (1+2)^{2}+(2+2)^{2}+(2+3)^{2}+5(2+4)^{2}+(3+4)^{2}+2(4+5)^{2} \\
& +2(4+6)^{2}+2(5+6)^{2}+3(3+6)^{2}+(3+3)^{2}+(5+3)^{2}+3(6+7)^{2} \\
& +2(6+6)^{2}+2(3+7)^{2}+3(4+7)^{2}+(4+4)^{2} \\
= & 2648 .
\end{aligned}
$$

Using the Definitions 3.1.3, 3.1.5, and Table 5, we have

$$
\begin{aligned}
R N M_{1}^{*}(G)= & \sum_{u v \in E(G)}\left[d_{N^{\prime}}(u)+d_{N^{\prime}}(v)\right] \\
= & (1+2)+(2+2)+(2+3)+5(2+4)+(3+4)+2(4+5)+2(4+6) \\
& +2(5+6)+3(3+6)+(3+3)+(5+3)+3(6+7)+2(6+6)+2(3+7) \\
& +3(4+7)+(4+4) \\
= & 274 . \\
R N M_{3}^{*}(G)= & \sum_{u v \in E(G)}\left[d_{N^{\prime}}(u)^{2}+d_{N^{\prime}}(v)^{2}\right] \\
= & \left(1^{2}+2^{2}\right)+\left(2^{2}+2^{2}\right)+\left(2^{2}+3^{2}\right)+5\left(2^{2}+4^{2}\right)+\left(3^{2}+4^{2}\right)+2\left(4^{2}+5\right) \\
+ & 2\left(4^{2}+6^{2}\right)+2\left(5^{2}+6^{2}\right)+3\left(3^{2}+6^{2}\right)+2\left(3^{2}+3^{2}\right)+\left(5^{2}+3^{2}\right)+3\left(6^{2}+7^{2}\right) \\
+ & 2\left(6^{2}+6^{2}\right)+2\left(3^{2}+7^{2}\right)+3\left(4^{2}+7^{2}\right)++\left(4^{2}+4^{2}\right) \\
= & 1388 .
\end{aligned}
$$

\section{Conclusions}

In this research work, some properties and calculations of the chemical compounds used for the treatment of COVID-19 in terms of reduced neighborhood topological indices are obtained. In particular, remdesivir, chloroquine, hydroxychloroquine, theaflavin, and dexamethasone. We evaluate the $R N M$ - polynomial of these structures with 3D graphical representation. Topological indices are very important to predict different properties and activities such as acentric factor, enthalpy, boiling point, critical pressure, entropy, etc. Our results and calculations may be useful in developing a new drug and vaccine to treat COVID19.

\section{Funding}

This research received no external funding. 


\section{Acknowledgments}

This research has no acknowledgment.

\section{Conflicts of Interest}

The authors declare no conflict of interest.

\section{References}

1. Wiener, H. Structural Determination of Paraffin Boiling Points. Journal of the American Chemical Society 1947, 69, 17-20, https://doi.org/10.1021/ja01193a005.

2. Katritzky, A.R.; Jain, R.; Lomaka, A.; Petrukhin, R.; Maran, U.; Karelson, M. Perspective on the Relationship between Melting Points and Chemical Structure. Crystal Growth \& Design 2001, 1, 261-265, https://doi.org/10.1021/cg010009s.

3. Gutman, I.; Trinajstić, N. Graph theory and molecular orbitals. Total $\varphi$-electron energy of alternant hydrocarbons. Chemical Physics Letters 1972, 17, 535-538, https://doi.org/10.1016/0009-2614(72)85099-1.

4. Wang, M.; Cao, R.; Zhang, L.; Yang, X.; Liu, J.; Xu, M.; Shi, Z.; Hu, Z.; Zhong, W.; Xiao, G. Remdesivir and chloroquine effectively inhibit the recently emerged novel coronavirus (2019-nCoV) in vitro. Cell Research 2020, 30, 269-271, https://doi.org/10.1038/s41422-020-0282-0.

5. Lung, J.; Lin, Y.-S.; Yang, Y.-H.; Chou, Y.-L.; Shu, L.-H.; Cheng, Y.-C.; Liu, H.T.; Wu, C.-Y. The potential chemical structure of anti-SARS-CoV-2 RNA-dependent RNA polymerase. Journal of Medical Virology 2020, 92, 693-697,https://doi.org/10.1002/jmv.25761.

6. The RECOVERY Collaborative Group. Dexamethasone in Hospitalized Patients with COVID-19 Preliminary Report. New England Journal of Medicine 2020, https://doi.org/10.1056/NEJMoa2021436.

7. Mondal, S.; De, N.; Pal, A. Topological Indices of Some Chemical Structures Applied for the Treatment of COVID-19 Patients. Promatic https://doi.org/10.1080/10406638.2020.1770306.

8. Kulli, V.R. Revan Indices of Chloroquine, Hydroxychloroquine, Remdesivir: Research Advances for the treatment of COVID-19. Int. J. Eng. Sci. Technol. 2020, 9, 73-74, https://doi.org/10.5281/zenodo.3828871.

9. Gao, W.; Siddiqui, M.K.; Imran, M.; Jamil, M.K.; Farahani, M.R. Forgotten topological index of chemical structure in drugs. Saudi Pharmaceutical Journal 2016, 24, 258-264, https://doi.org/10.1016/j.jsps.2016.04.012.

10. Mondal, S.; De, N.; Pal, A. Multiplicative Degree Based Topological Indices of Nanostar Dendrimer. Biointerface Res. Appl. Chem. 2021, 11, 7700-7711, https://doi.org/10.33263/BRIAC111.77007711.

11. Mondal, S.; De, N.; Pal, A. On Some General Neighborhood Degree Based Topological Indices. International Journal of Applied Mathematics 2019, 32, 1037-1049, https://doi.org/10.12732/ijam.v32i6.10.

12. Mondal, S.; De, N.; Pal, A. Topological Properties of Networks Using M-Polynomial Approach. Konuralp J. Math. 2020, 8, 97-105

13. Sunilkumar, M.H. Quantitative Structure Property Analysis of Anti-COVID-19 Drugs. ArXiv:2008.07350. 2020.

14. Afzal, F.; Hussain, S.; Afzal, D.; Razaq, S. Some new degree based topological indices via M-polynomial. Journal of Information and Optimization Sciences 2020, 41, 1061-1076, https://doi.org/10.1080/02522667.2020.1744307.

15. Mondal Sourav, De Nilanjan, and Pal Anita, “QSPR Analysis of Some Novel Neighborhood Degree Based Topological Descriptors," arXiv:1906.06660 2019.

16. Shafiei, F. Relationship between Topological Indices and Thermodynamic Properties and of the Monocarboxylic Acids Applications in QSPR. Iranian J. Math. Chem. 2015, 20, 15-28.

17. Tavakoli, M.; Yousefi-Azari, H. Computing PI and Hyper-Wiener Indices of Corona Product of some Graphs. Iranian J. Math. Chem. 2010, 1, 131-135, https://dx.doi.org/10.22052/ijmc.2010.5144.

18. Mondal, S.; De, N.; Pal, A. Topological properties of Graphene using some novelneighborhood degree-based topological indices. Int. J. Ind. Math. 2020, 11, https://doi.org/10.1142/S2661335219500060.

19. Mondal, S.; Siddiqui, M.K.; De, N.; Pal, A. Neighborhood M-polynomial of crystallographic structures Biointerface Res. Appl. Chem. 2021, 11, 9372-9381, https://doi.org/ 10.33263/BRIAC112.93729381.

20. Liu, J.B.; Zhao, J.; He, H.; Shao, Z. Valency-Based Topological Descriptors and Structural Property of the Generalized Sierpinski Networks. J. Stat. Phys. 2019, 177, 1131-1147, https://doi.org/10.1007/s10955-01902412-2. 